\title{
Spinal shock: possible role of receptor plasticity and non synaptic transmission
}

\author{
P Bach-y-Rita MD, ${ }^{1}$ L S Illis MD BSc FRCP ${ }^{2}$ \\ ${ }^{1}$ University of Wisconsin-Madison Medical School, 1300 University Avenue, Room 2756, \\ Madison WI53706, USA; ${ }^{2}$ Wessex Neurological Centre, Southampton University Hospitals \\ (Southampton General Hospital), Southampton, England.
}

\begin{abstract}
Spinal shock remains an enigma. To date there has been no convincing explanation of the recovery of reflexes following their complete abolition. Volume transmission includes both the activation of extrasynaptic receptors, and activity induced by substances diffusing into synaptic clefts via the extracellular fluid. A brief review of non synaptic transmission is given, and a review of spinal shock. We suggest that the recovery of reflexes in spinal shock may be related to the up regulation of receptors, resulting in increased sensitivity to neurotransmitters and other neuroactive substances released at the surviving synapses, or elsewhere, and transported in the extracellular fluid.

Further understanding of spinal shock would give both practical help for the patient and have academic implications for the scientific basis of neurological rehabilitation.
\end{abstract}

Keywords: spinal shock; receptor plasticity; volume transmission.

\section{Introduction}

Spinal shock remains an enigma which challenges the neurophysiologist and the clinical neurologist; its solution would both give practical help for the patient and have academic implications for the scientific basis of neurological rehabilitation.

Spinal shock is the term used to signify the effect of sudden injury or transection of the spinal cord. It is characterised by sensory and motor loss and, later, gradual recovery of reflexes. To date there has not been a convincing explanation of the recovery of reflexes following their complete abolition. We suggest in this speculative synthesis that the recovery of reflexes may be related to the up regulation of receptors in synapses and on the surface of partially denervated spinal cord cells, resulting in increased sensitivity to neurotransmitters and other neuroactive substances released at the surviving synapses or elsewhere and transported in the extracellular fluid. This leads to non synaptic neurotransmission.

\section{Non synaptic transmission}

Non synaptic (or extrasynaptic) transmission has also been called volume transmission, a term proposed by Fuxe and Agnati, ${ }^{1}$ for electrical and chemical communication in the brain via the extracellular fluid. It can include both the activation of extrasynaptic receptors, and the activity induced by substances diffusing into synaptic clefts from the extracellular fluid. Changeux ${ }^{2}$ considers that volume transmission is a '... complementary, if not alternative, mechanism to classical chemical synaptic transmission'. He noted that the concept proposes that in the nervous system signalling may take place via extracellular spaces as well as via synapses. In volume transmission, neurotransmitters and other neuroactive substances are released at points that may be remote from the target cells, which they reach by diffusion through the extracellular fluid to exert desynchronised and sustained influences on vast neuronal ensembles.

Bach-y-Rita ${ }^{3}$ recorded sustained activity 
in brain cells following stimulation of several modalities from various parts of the body, and proposed a neurohumoral transmission mechanism by local diffusion. However, further elaboration of the concept could not occur until receptor identification methods were developed. Volume transmission has been implicated in information transmission, ${ }^{4,5}$ in reorganisation of function following brain damage, and in the development of a scientific basis for neurological rehabilitation. ${ }^{6-9}$

The evidence for mismatches between neurotransmitter and receptor localisations has recently been evaluated by Herkenham. ${ }^{10} \mathrm{He}$ noted that mismatches had previously been considered exceptional occurrences, but he now considers them to be the rule rather than the exception. He noted that the mismatches occur in peptide, monoamine and amino acid systems. Transmitter release distant from receptor localisation suggests that volume transmission is an important mechanism of neuronal communication.

Descarries et al $^{11}$ have confirmed that the monoamine systems in the brain, including serotonin, dopamine and noradrenaline, are highly non synaptic. Their immunocytochemical studies confirmed the autoradiograph findings of Beaudet and Descarries ${ }^{12}$ which also characterised the noradrenaline system as mostly non junctional.

The intercellular space comprises approximately $20 \%$ of the volume of the living brain. ${ }^{13}$ It is ample for many kinds of neuroactive substances to diffuse from release points to receptors on the neurons of the cerebral cortex. Among the neuroactive substances found in the extracellular fluid are dopamine, choline, adenosine, noradrenalin, serotonin, acetylcholine, and others. ${ }^{14,15}$ They may diffuse into synaptic clefts, and receptors on the surface of the cell can also be contacted by, and bound to, the neurotransmitter and other ligands diffusing in the extracellular fluid. Fuxe and Agnati ${ }^{1}$ pointed out that the structure of the brain cell microenvironment may depend on physical and chemical constraints represented by cellular elements such as astroglia, which also have been shown to have receptors for.various neurotransmitters and other neuroactive substances. ${ }^{16}$ The density of boutons termineaux on the motor neuron surface ranges from $50-69 \% .{ }^{17}$ That is the total surface area taken up by synaptic contacts. The remaining $31-50 \%$ of the cell surface is either bare or in contact with glial cells and their processes. Synaptic 'bare areas' are essential for the presence of postsynaptic potentials.

Bach-y-Rita ${ }^{4,7}$ has speculated on the role of volume transmission in mediating mass, sustained functions such as sleep and vigilance, and mood. The widespread activation of groups of neurons via diffusion should be more energy-efficient than synaptic activation of those same neurons. When a muscle fibre is denervated, there follows a massive up regulation of receptors on the entire cell membrane, resulting in hypersensitivity to low concentrations of acetycholine. ${ }^{18}$ Bach$\mathrm{y}$-Rita ${ }^{4}$ has suggested that if up regulation of specific receptors has occurred following brain damage resulting in partial or total denervation (comparable to the massive up regulation on the cellular membrane of denervated muscle), those cells may similarly become hypersensitive to specific neuroactive substances, and thus respond to the low concentrations of those substances in the extracellular fluid. Other examples of receptor up regulation and hypersensitivity, possibly including drug addiction and kindling, may be studied in the context of volume transmission in the future.

Receptor changes indicate not only the response of denervated neural tissue, but the reaction of undamaged tissue to partial denervation. Volume transmission may have particular relevance to the production of activity in either partially denervated neurons or in undamaged neurons relatively distant from the site of injury. Volume transmission, rather than conventional synaptic neurotransmission may be of more relevance to the way in which the nervous system reacts to a specific injury. Spinal shock is perhaps the most well known and most extreme form of neurological damage and is still very poorly understood. The term 'spinal shock' was introduced by Marshall Hall $^{19}$ although the actual phenomena were described earlier by Whytt. ${ }^{2}$ Any theory which attempts to explain the phenomena of 
spinal shock must include the recovery of reflexes.

\section{Spinal shock}

Spinal shock occurs at any level of transection below the mid pons. Above this level a transection produces decerebrate rigidity. Following transection below the pons, nerve cells in the spinal cord are suddenly cut off from all descending influences and then undergo a dramatic change in their capacity to react. The relative importance of different pathways in producing spinal shock is poorly known, but in lower animals the important descending influences appear to be reticulospinal and vestibulospinal, whereas in higher animals, including man, corticospinal connections are probably more important. Although it is usually assumed that the importance of the pathways in producing spinal shock is a reflection of their specificity, one can equally argue that their importance lies in their relative contribution to the total spinal cord input. The fact that the depression of spinal shock is much more severe when transection is below the pons does not necessarily mean that there is some particularly important control arising in pontine nuclei; it could mean that the difference in transection above and below the pons is due to the relative contribution to the descending pathways made by different levels of the neuraxis. That is, the greater the number of descending pathways interrupted the more likely that shock will ensue because the more severely disorganised will be the area upon which these descending pathways converge and the more likely that they will produce a critical alteration in receptor response (see below). A higher contribution of inputs from a particular centre does, of course, confer some specificity, but it is not necessary to assign the capacity for production of spinal shock on to a particular centre or its pathway.

There are many detailed descriptions of spinal shock and it is not necessary to reiterate them all here. All are illustrations rather than explanations of spinal shock. It is clear that the cause of the first phase of spinal shock is far from being explained by withdrawal of impulses from above, since the sudden withdrawal of facilitation decreases the reflexes more than would be accounted for by a mere withdrawal of an excitatory background and the second phase (return of reflexes in an altered form) is similarly not explained by withdrawal of higher control.

There are two further features of spinal shock which are of particular interest. First, it has been commonly assumed that the effects of transection occur in an aboral direction only. This is not so, since headward of the transection there is a change in the reflex response. ${ }^{21}$ Secondly, Teasdell and Stavraky ${ }^{22}$ described deafferentation of one limb of a cat by section of the posterior roots; electrical stimulation of the basis peduncli produced no response in the corresponding limb, but 5-47 days later responses were evoked more readily in the denervated than in the normal limb. This is a comparable state of affairs to that pertaining to spinal shock and has particular relevance to theories of spinal shock, ${ }^{23}$ especially those in which volume transmission and receptor plasticity may play a role.

Transection of the spinal cord produces dramatic changes in cord function. A severe spinal lesion, short of complete transection, results in a series of vascular and biochemical changes which have a bearing on synaptic and receptor function. These changes include alteration in blood flow, summarised by Young. ${ }^{24}$ Within several hours of a severe contusion, gross haemorrhagic necrosis develops in the grey matter with evidence of endothelial breakdown and coagulation leading to ischaemia and progressive tissue damage. Blood flow in the grey matter falls rapidly but in the white matter blood flow changes are delayed by several hours. Intracellular calcium ionic activity is normally extremely low but with injury calcium ions rapidly move in to injured nerve cells and affect cellular transport and synaptic transmission. In addition, elevation of calcium ions activates phospholipases which in turn leads to membrane breakdown. At the same time traumatic injury disrupts cells and releases potassium and neurotransmitters into the extracellular 
space and this will affect calcium influx into neighbouring nerve cells.

Experimental work has suggested that at the motor neuron surface there is an extremely complex mosaic of nerve terminals from various sources intermixed with glial cells. The nerve cell surface is not simply the site of termination of a few hundred boutons termineaux. On a large nerve cell such as the anterior horn cell in a cat spinal cord, the motor neuron surface has up to 30,000 synapses. ${ }^{17,25}$ These endings occupy up to $70 \%$ of cell and dendrite surface and are present on the dendrite as far out as they can be traced from the cell body. The synapses clothe the cell surface like a mosaic, the constituent parts of which are separated by bare areas and by glial cells and their processes. Within the area encompassed by one glial cell and its processes there are inputs from different sources since the boutons of any incoming nerve fibre are widely scattered over the cell surface. This zone, consisting of boutons termineaux, postsynaptic thickenings, glial cells and their processes may be called the 'synaptic zone' ${ }^{25}$ If the steady state of the synaptic zone is disturbed by a partial denervation then synapses which have had their afferent fibres cut will degenerate, but neighbouring synapses whose afferent fibres were intact show a temporary reversible change (disorganisation) which is most marked at 24 hours. ${ }^{17,23}$ The widespread reversible change depends on a critical number of synapses being denervated. For example, when posterior roots are cut on one side of the spinal cord, the disorganisation is much more marked on the ipsilateral than on the contralateral side. If the motor cortex of the cat is ablated and then changes are looked for in the spinal cord, the changes of disorganisation seen at 24 hours are either absent or only doubtfully present although the changes of degeneration are seen. This is presumably due to a difference in density of endings on target nerve cells so that if less than the critical number of synapses are denervated then no disorganisation takes place. This disorganisation is indicated by alteration in staining characteristics as well as by physiological changes. The alteration in staining characteristics has been shown to be due to changes associated with synaptic vesicles. ${ }^{26}$

What is the relevance of these histological changes to the phenomena of spinal shock? Valtschanoff et al ${ }^{27}$ carried out experiments labelling with WGA-HRP after unilateral nerve crush or transection in rats, and demonstrated increased release of the tracer into extracellular spaces. The density of labelling was similar to the disorganisation seen in histological studies mentioned above. This suggests that endogenous substances may be released into these spaces and act as a vehicle for signalling to a wide population of neurons served by the central processes of the injured axons and possibly mediate slow neuronal processes producing an effect much more widespread than expected from conventional methods of tracing central pathways.

Theories relating to the nature of the return of reflexes in the second phase of spinal shock must bear upon two further great neurological problems: the capacity of the nervous system to learn, and the capacity of the central nervous system to regenerate or reorganise. One of the important features of recovery after spinal shock is not simply the presence of abnormal reflexes and the time sequence of their return, but also the demonstration of an altered receptive field so that reflexes may be elicited by minimal stimulation of the skin from a progressively wider area. This enlargement of the receptive field is perhaps the most obvious evidence of alteration in the intact nervous system in response to a lesion. As the first stage of spinal shock subsides, afferent impulses from the periphery begin to elicit an excitatory influence. Reflexes return in a sequential way and not simultaneously. During the early return of reflexes the stimulus must be strong or summated and the response shows easy fatigue. Following cord section in the cat, reflexes below the lesion are obtainable after about one hour. But not all reflexes are so readily obtainable, and over a long period of time reflexes are returning and are still changing in their nature. They come back in the order: knee jerk, crossed extensor and extensor thrust; the last to appear is the scratch reflex. Sherrington ${ }^{28}$ observed that 
the scratch reflex 6 weeks after cord section was still irregular, feeble and easily fatigued and this was confirmed by Fulton and Sherrington. ${ }^{29}$ Despite the variability in reflex pattern and the variability in timing of return, there are certain dominant patterns of reflex return. All these abnormal reflex patterns have one thing in common: the stimulus elicits not the usual response, ie the response expected in the intact organism, but an abnormal pattern not seen in the undamaged spinal cord. The abnormal pattern of reflexes may reflect selective receptor up and/or down regulation and neurotransmission via the extracellular fluid, or may be a reflection of previously existing anatomical pathways whose function is normally masked; ${ }^{30}$ ie these pathways, in the intact nervous system, are structurally intact but play little or no functional part until degeneration of more significant pathways has occurred. Does the unmasking occur simply through the removal of descending control (whatever that precisely means), through chemical hypersensitivity of undamaged endings, or through sprouting of new terminals? This remains a matter of argument and of interpretation but the study of volume transmission brings even further interpretations.

The abnormal reflex patterns may represent, in addition, a central alteration of receptive fields (possibly related to receptor plasticity) secondary to an alteration in afferent input and this itself may be a reflection of partial paralysis. For example, although the neurological syndrome may be primarily motor such as a paralysed limb, the effect on the central nervous system is a deficit of sensory information, and not a deficit of motor impulses.

\section{Conclusion}

The studies reviewed above describe non synaptic transmission, a mechanism for neurotransmission by means of the diffusion of neurotransmitters and other neuroactive substances in the extracellular fluid, with the resulting activation of synaptic and extrasynaptic receptors on the cells. Also reviewed are studies that suggest that a lesion producing partial or complete denervation leads to the up and/or down regulation of receptors both at synapses and on the extrasynaptic membrane, and thus to hypersensitivity to neuroactive substances. We consider it possible that similar mechanisms may relate to spinal shock, with the recovery of reflexes. Our principal purpose has been to stimulate new approaches, both conceptual and experimental, to an understanding of spinal shock, and to the establishment of a scientific basis for neurological rehabilitation.

\section{References}

1 Fuxe K, Agnati LF (1991) Two principal modes of electrochemical communication in the brain: volume versus wiring transmission. In: Fuxe $\mathrm{K}$, Agnati LF, editors. Volume Transmission in the Brain. Raven Press, New York: 1-9.

2 Changeux JP (1991) Concluding remarks. In: Fuxe K, Agnati LF, editors. Volume Transmission in the Brain. Raven Press, New York: 569-585.

3 Bach-y-Rita P (1964) Convergent and long latency unit responses in the reticular formation of the cat. Exp Neurol 9: 327-344.

4 Bach-y-Rita P (1991) Thoughts on the role of volume transmission in normal and abnormal mass sustained functions. In: Fuxe K, Agnati LF, editors. Volume Transmission in the Brain. Raven Press, New York: 489-496.

5 Bach-y-Rita $P$ (in press) Volume (extrasynaptic) transmission. In: Adelman G, Smith B, editors. Neuroscience Year; Supplement 3 to the Encyclopedia of Neuroscience. Birkhauser, Boston.

6 Bach-y-Rita P (1990) Receptor plasticity and volume transmission in the brain: emerging concepts with relevance to neurologic rehabilitation. J Neurol Rehabil 4: 121-128.

7 Bach-y-Rita P (in press) Volume transmission in the brain, sensory substitution, and home stroke rehabilitation: emerging brain plasticity-related concepts in neurologic rehabilitation. In: Illis LS, editor. Neurological Rehabilitation. Blackwells, Oxford.

8 Bach-y-Rita P, Bjelke B (1991) Lasting recovery of motor function, following brain damage, with a single dose of amphetamine combined with physical therapy; changes in gene expression? Scand J Rehabil Med 23: 219-220. 
9 Bach-y-Rita P, Wicab Bach-y-Rita E (1990) Biological and psychosocial factors in recovery from brain damage in humans. Can J Psychol 44: 148-165.

10 Herkenham M (1991) Mismatches between neurotransmitter and receptor localisations: implications for endocrine functions in brain. In: Fuxe K, Agnati LF, editors. Volume Transmission in the Brain. Raven Press, New York: 63-87.

11 Descarries L, Seguela P, and Watkins KC (1991) Nonjunctional relationships on monoamine axon terminals in the cerebral cortex of adult rat. In: Fuxe K, Agnati LF, editors. Volume Transmission in the Brain. Raven Press, New York: 53-62.

12 Beaudet A, Descarries L (1978) The monoamine innervation of rat cerebral cortex: synaptic and nonsynaptic axon terminals. Neurosci 3: 851-860.

13 Nicholson C, and Rice ME (1991) Diffusion of ions and transmitters in the brain cell microenvironment. In: Fuxe K, Agnati L, editors. Volume Transmission in the Brain. Raven, New York.

14 Ungerstedt U (1984) Measurement of neurotransmitter release by intracranial dialysis. In: Marsden CA, editor. Measurement of Neurotransmitter Release in Vivo. John Wiley \& Sons Ltd, Chichester: 81-105.

15 Di Chiara G (1990) In-vivo brain dialysis of neurotransmitters. Trends Pharmacol Sci 11: 116-121.

16 Hansson E (1991) Transmitter receptors on astroglial cells. In: Fuxe K, Agnati LF, editors. Volume Transmission in the Brain. Raven Press, New York: 257-265.

17 Illis LS (1964) Spinal cord synapses in the cat: the normal appearances by the light microscope. Brain 87: 543-554.

18 Salpeter MM, Loring RH (1985) Nicotinic acetylcholine receptors in vertebrate muscle properties, distribution and neural control. Progr Neurobiol 25: 297-325.

19 Marshall Hall (1850) Synopsis of the Diastaltic Nervous System. London. Quoted by Sherrington (1906): 241.

20 Whytt R (1750) Quoted by Sherrington (1906): 241.

21 Creed RS, Denny-Brown D, Eccles JC, Liddell EGT, Sherrington CS (1932) Reflex Activity of the Spinal Cord. Oxford University Press, London.

22 Teasdell RD, Stavraky GW (1953) Responses of deafferented spinal neurones to corticospinal impulses. $J$ Neurophysiol 16: 367-375.

23 Illis LS (1963) Changes in spinal cord synapses and a possible explanation for spinal shock. Exp Neurol 8: $328-335$.

24 Young W (1992) Therapy of acute spinal injury. In: Illis LS, editor. Spinal Cord Dysfunction II: Intervention and Treatment. Oxford University Press.

25 Illis LS (1967) Modern trends in neurology. In: Williams D, editor. The Motoneurone Surface and Spinal Shock. Butterworths, London.

26 Fitzsimons JTR, Illis LS, Mitchell J (1974) Silver deposition in synaptic vesicles using the ArmstrongStephens stain. Brain Res 72: 277-281.

27 Valtschanoff JG, Weinberg RJ, Rustioni A (in press) Peripheral injury and anterograde transport of WGA-HRP to the spinal cord. Neuroscience 50: 685-696.

28 Sherrington CS (1906) The Integrative Action of the Nervous System. Charles Scribner's Sons, New York.

29 Fulton JF, Sherrington CS (1932) State of the flexor reflex in paraplegic dog and monkey respectively. $J$ Physiology (Lond.) 75: 17-22.

30 Merrill EG, Wall PD (1978) Plasticity of connections in the adult nervous system. In: Cotman CW, editor. Neuronal Plasticity. Raven Press, New York: 97-111. 